\title{
COLLINEATIONS IN WEYL SPACES OF TWO DIMENSIONS
}

\author{
JACK LEVINE
}

1. Introduction. In this paper we obtain all (real) two-dimensional Weyl spaces $\left(W_{2}\right)$ which admit (real) continuous groups $G_{r}$ of affine collineations (A.C.). Such a collineation is defined as a point transformation which changes each path of the space into a path and also preserves the affine path-parameters $\left[1\right.$, p. 126]. ${ }^{1}$ The work is based on the results of another paper to be published elsewhere [2]. It is shown in [2] that there are 12 types of affinely connected spaces $A_{2}$ admitting A.C. When each of these types is tested for reducibility to a $W_{2}$ it is found that all but three are eliminated, these three admitting respectively A.C. of one, two, and three parameters. There are four general types of $W_{2}$ admitting the $G_{1}$ of A.C. and also four such types of $W_{2}$ admitting the $G_{2}$ of collineations. Corresponding to the $G_{3}$ there is only one class of $W_{2}$.

2. Weyl spaces. A Weyl space $W_{n}$ is a special type of affine space $A_{n}$ (symmetric connection) which admits a tensor and a covariant vector with components $g_{i j}, \phi_{i}$ respectively such that

$$
g_{i j, k}+2 g_{i j} \phi_{k}=0,
$$

where a comma denotes covariant differentiation with respect to the components $\Gamma_{j k}^{i}$ of affine connection of the space $[1, \mathrm{p} .81]$. The $g_{i j}$ are assumed symmetric. In addition the vector $\phi_{i}$ must not be a gradient (as otherwise the $W_{n}$ reduces to a Riemannian space) and we must have also

$$
\left|g_{i i}\right| \neq 0 \text {. }
$$

The $\Gamma_{j k}^{\mathfrak{k}}$ can be expressed as

$$
\Gamma_{j k}^{i}=\left\{\begin{array}{c}
i \\
j k
\end{array}\right\}+\delta_{j}^{i} \phi_{k}+\delta_{k}^{i} \phi_{j}-g_{i k} g^{i m} \phi_{m} .
$$

The components $g_{i j}, \phi_{i}$ of a $W_{n}$ are determined to within an arbitrary function $\theta\left(x^{i}\right)$, that is, the tensors with components $\bar{g}_{i j}$, $\bar{\phi}_{i}$ given by

$$
\bar{g}_{i j}=e^{2 \theta} g_{i j},
$$

$$
\bar{\phi}_{i}=\phi_{i}-\partial \theta / \partial x^{i}
$$

will also satisfy (2.1). The $\Gamma_{j k}^{d}$ remain invariant under (2.4) which is

Received by the editors February 15, 1950.

${ }^{1}$ Numbers in brackets refer to the bibliography at the end of the paper. 
the change of gauge of Weyl. When a definite $\theta$ is picked the space is said to be calibrated [3].

Now the conditions of integrability of (2.1) are

$$
g_{h j} B_{i k m}^{h}+g_{i h} B_{j k m}^{h}+2 g_{i j}\left(\phi_{k, m}-\phi_{m, k}\right)=0,
$$

from which it follows that

$$
B_{i k m}^{i}=2\left(\frac{\partial \phi_{m}}{\partial x^{k}}-\frac{\partial \phi_{k}}{\partial x^{m}}\right) \quad(n=2),
$$

where the curvature tensor components $B_{j k m}^{i}$ are

$$
B_{j k m}^{i}=\frac{\partial \Gamma_{j m}^{i}}{\partial x^{k}}-\frac{\partial \Gamma_{j k}^{i}}{\partial x^{m}}+\Gamma_{j m}^{h} \Gamma_{h k}^{i}-\Gamma_{j k}^{h} \Gamma_{h m}^{i} .
$$

It follows that we must have $(n=2)$

$$
B_{i 12}^{i}=\frac{\partial \Gamma_{i 2}^{i}}{\partial x^{1}}-\frac{\partial \Gamma_{i 1}^{i}}{\partial x^{2}} \neq 0 .
$$

If then we apply the test (2.8) to the 12 types of $A_{2}$ obtained in [2]. we find that the only types not eliminated are

(I) $\Gamma_{j k}^{i}=\Gamma_{j k}^{i}(y)$, with $G_{1}=[p]$,

(II) $\Gamma_{j k}^{i}=(1 / y) a_{j k}^{i}$, with $G_{2}=[p, x p+y q]$,

(III) $\Gamma_{11}^{1}=2 b / y^{2}, \quad \Gamma_{12}^{1}=-1 / y, \quad \Gamma_{22}^{1}=0, \quad \Gamma_{11}^{2}=a / y^{3}, \quad \Gamma_{12}^{2}=b / y^{2}, \quad \Gamma_{22}^{2}$ $=-2 / y$, with $G_{3}=\left[p, 2 x p+y q, x^{2} p+x y q\right]$.

In (I, II, III) we have used the notation (to be followed hereafter) $x, y$ for $x^{1}, x^{2}$, and also $p=\partial f / \partial x, q=\partial f / \partial y$. The infinitesimal generators $X_{\alpha} f=\xi_{\alpha \mid}^{1} p+\xi_{\alpha \mid}^{2} q \quad(\alpha=1, \cdots, r)$ will generate the $G_{r}$ $=\left[X_{1}, X_{2}, \cdots, X_{r}\right]$ (in the neighborhood of the identity transformation).

The $\Gamma_{j k}^{i}(y)$ in (I) are arbitrary functions of $y$. In (II), $a_{j k}^{i}$ are arbitrary constants, as also are $a, b$ in (III). These three cases (I), (II), (III) are the $[1.1],[2.1],[3.1]$ respectively of [2].

The equations (2.5) of integrability can be reduced by means of (2.6) to the set of (dependent) equations

$$
\begin{aligned}
& \left(B_{12}+B_{21}\right) g_{11}-2 B_{11} g_{12}=0 \text {, } \\
& 2 B_{22} g_{12}-\left(B_{12}+B_{21}\right) g_{22}=0 \text {, } \\
& B_{22} g_{11} \\
& -B_{11} g_{22}=0 \text {. }
\end{aligned}
$$

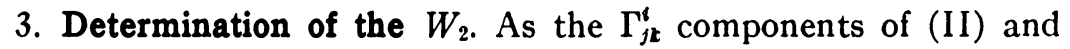
(III) are special cases of those of (I), it is sufficient to obtain the 
solutions for this latter case and then by suitable specializations obtain those for (II) and (III).

We first observe that from (2.4b) and (2.6) there is no loss of generality in assuming

$$
\phi_{1}=\left(\Gamma_{11}^{1}+\Gamma_{12}^{2}\right) / 2, \quad \phi_{2}=0 .
$$

Since if $B_{i j}$ be skew-symmetric integrability conditions (2.9) are satisfied identically, it is convenient to consider two cases according as $B_{i j}$ is or is not skew. In case $B_{i j}$ is not skew we consider further the two subcases I(a) $B_{11} \neq 0, \mathrm{I}$ (b) $B_{11}=0$.

If $B_{11} \neq 0$, we can find a coordinate transformation of the type

$$
x=\bar{x}+f(\bar{y}), \quad y=\bar{y},
$$

such that $\bar{B}_{12}+\bar{B}_{21}=0, \bar{B}_{11} \neq 0$, and also have the $\bar{\Gamma}_{j \mathrm{k}}^{i}$ as functions of $\bar{y}$ only. By a suitable change of gauge conditions (3.1) will hold in the barred coordinates also. If then the bars be dropped, we have from (2.9) and (2.2) that $g_{12}=0, B_{22} \neq 0$.

Equations (2.1) when expanded are (using (3.1))

$$
\begin{aligned}
& \frac{\partial g_{11}}{\partial x}=g_{11}\left(\Gamma_{11}^{1}-\Gamma_{12}^{2}\right)+2 g_{12} \Gamma_{11}^{2}, \\
& \frac{\partial g_{11}}{\partial y}=2 g_{11} \Gamma_{12}^{1}+2 g_{12} \Gamma_{12}^{2}, \\
& \frac{\partial g_{12}}{\partial x}=g_{11} \Gamma_{12}^{1}+g_{22} \Gamma_{11}^{2}, \\
& \frac{\partial g_{12}}{\partial y}=g_{11} \Gamma_{22}^{1}+g_{12}\left(\Gamma_{12}^{1}+\Gamma_{22}^{2}\right)+g_{22} \Gamma_{12}^{2}, \\
& \frac{\partial g_{22}}{\partial x}=2 g_{12} \Gamma_{12}^{1}-g_{22}\left(\Gamma_{11}^{1}-\Gamma_{12}^{2}\right), \\
& \frac{\partial g_{22}}{\partial y}=2 g_{12} \Gamma_{22}^{1}+2 g_{22} \Gamma_{22}^{2} .
\end{aligned}
$$

The solutions of (3.3) corresponding to case $I(a)$ are given in $\$ 4$. In the subcase $I(b)$ for which $B_{11}=0$, we must have $B_{12}+B_{21} \neq 0$, for if not (2.9) shows $B_{22}=0$, that is, $B_{i j}$ is skew.

By a (3.2) type transformation we can make $B_{22}=0$, and (3.3) can be easily solved. The solutions are given below.

If $B_{i j}$ is skew we again assume two subcases: $\mathrm{I}(\mathrm{c}) \Gamma_{11}^{2} \neq 0, \mathrm{I}(\mathrm{d}) \Gamma_{11}^{2}$ $=0$. In $\mathrm{I}(\mathrm{c})$ we can make $\Gamma_{12}^{2}=0$ by (3.2) (still keeping $\Gamma_{11}^{2} \neq 0$ ). In 
$\mathrm{I}(\mathrm{d}),(3.2)$ is used to make $\Gamma_{22}^{1}=0$. In addition, it is easily shown that we must have $\Gamma_{12}^{1}=0, \Gamma_{12}^{2}=\Gamma_{11}^{1} \neq 0$.

The solutions of (3.3) corresponding to (II) are given as II(a)II(d). The form of the $G_{2}$ remains invariant under (3.2).

In dealing with (III) we first reduce it to the case $\mathrm{I}(\mathrm{c})$ of (I) by a (3.2) transformation. Here we must have $4 a-b^{2}=0, b \neq 0$. The solution and transformed form of the $G_{3}$ are given in III(a).

It can be shown as in [2] that the three groups are complete groups of affine collineations.

\section{The solutions.}

$W_{2}$ admitting the one-parameter group $G_{1}=[p]$

I(a). $B_{i j}$ now skew, $B_{11} B_{22} \neq 0, B_{12}+B_{21}=0$.

$$
\begin{array}{rlrl}
\Gamma_{11}^{1} & =\Gamma_{12}^{2} \neq 0, \quad \Gamma_{11}^{\prime 2} \neq 0, \quad \Gamma_{11}^{1} \Gamma_{12}^{1}-\Gamma_{22}^{1} \Gamma_{11}^{2}=0, & \\
\left(B_{22} / B_{11}\right)^{\prime} & =2\left(B_{22} / B_{11}\right)\left(\Gamma_{22}^{2}-\Gamma_{12}^{1}\right), & \\
g_{11} & =a \exp \left(2 \int \Gamma_{12}^{1} d y\right), \quad g_{12}=0, \\
g_{22} & =b \exp \left(2 \int \Gamma_{22}^{2} d y\right)=\frac{B_{22}}{B_{11}} g_{11} \\
\phi_{1} & =\Gamma_{11}^{1}, \quad \phi_{2}=0 .
\end{array}
$$

I(b). $B_{i j}$ not skew, $B_{11}=B_{22}=0, B_{12}+B_{21} \neq 0$.

$$
\begin{aligned}
& \Gamma_{11}^{1}=\Gamma_{12}^{1}=\Gamma_{11}^{2}=\Gamma_{12}^{2}=0, \quad \Gamma_{11}^{\prime 1}=0, \\
& g_{11}=0, \quad g_{12}=a \exp \left(\int \Gamma_{22}^{2} d y\right), \quad g_{22}=0 \quad(a \neq 0), \\
& \phi_{1}=\Gamma_{11}^{1} / 2, \quad \phi_{2}=0 .
\end{aligned}
$$

I(c). $B_{i j}$ skew.

$$
\begin{aligned}
\Gamma_{11}^{2} & \neq 0, \quad \Gamma_{11}^{\prime 1} \neq 0, \quad \Gamma_{12}^{2}=0 ; \quad \phi_{1}=\Gamma_{11}^{1} / 2, \quad \phi_{2}=0 \\
g_{11} & =g(x) F(y), \quad g_{12}=\frac{F}{2 \Gamma_{11}^{2}}\left(g^{\prime}-g \Gamma_{11}^{1}\right), \\
g_{22} & =\frac{F}{2\left(\Gamma_{11}^{2}\right)^{2}}\left(g^{\prime \prime}-g^{\prime} \Gamma_{11}^{1}-2 g \Gamma_{12}^{1} \Gamma_{11}^{2}\right), \\
F(y) & =\exp \left(2 \int \Gamma_{12}^{1} d y\right)
\end{aligned}
$$




$$
\begin{gathered}
g(x)=a x^{2}+b x+c, \quad b^{2}-4 a c \neq 0, \quad\left(\Gamma_{11}^{1}\right)^{2}+4 \Gamma_{12}^{1} \Gamma_{11}^{2}=0, \\
g(x)=a e^{k x}+b e^{-k x}+c, \quad c^{2}-4 a b \neq 0, \quad\left(\Gamma_{11}^{1}\right)^{2}+4 \Gamma_{12}^{1} \Gamma_{11}^{2}=k^{2}, \\
g(x)=a \cos k x+b \sin k x+c, \quad c^{2}-a^{2}-b^{2} \neq 0, \\
\left(\Gamma_{11}^{1}\right)^{2}+4 \Gamma_{12}^{1} \Gamma_{11}^{2}=-k^{2} .
\end{gathered}
$$

I(d). $B_{i j}$ skew.

$$
\begin{aligned}
\Gamma_{12}^{1} & =\Gamma_{11}^{2}=\Gamma_{11}^{1}-\Gamma_{12}^{2}=0, \Gamma_{11}^{\prime 1} \neq 0 ; \phi_{1}=\Gamma_{11}^{1}, \phi_{2}=0 \\
g_{11}(y) & =\int 2 g_{12} \Gamma_{11}^{1} d y, \quad g_{12}=\int g_{22} \Gamma_{11}^{1} d y \\
g_{22} & =a \exp \left(\int 2 \Gamma_{22}^{2} d y\right)
\end{aligned}
$$

In all cases the $\Gamma_{j \mathbf{k}}^{\mathbf{k}}$ are understood to be arbitrary functions of $y$ subject to indicated restrictions.

$W_{2}$ admitting the two-parameter group $G_{2}=[p, x p+y q]$

$\Gamma_{j k}^{i}=(1 / y) a_{j \mathbf{k}}^{i}$ throughout, $a_{j k}^{i}$ arbitrary constants subject to indicated restrictions.

II(a). $\quad a_{11}^{1}=\stackrel{2}{a_{12}} \neq 0, \quad a_{12}^{1}=a_{22}^{2} \neq 0$,

$$
\begin{aligned}
& a_{11}^{2} \neq 0, \quad 1+a_{12}^{1}-a_{11}^{2} \neq 0, \quad a_{22}^{1} a_{11}^{2}-a_{12}^{1} a_{11}^{1}=0, \\
& \phi_{1}=\frac{1}{y} a_{11}^{1}, \quad \phi_{2}=0, \\
& g_{11}=a y^{2 a_{12}^{1}}, g_{12}=0, g_{22}=b y^{2 a_{12}^{1}}(a b \neq 0), a a_{12}^{1}+b a_{11}^{2}=0 .
\end{aligned}
$$

II(b). $\quad a_{12}^{1}=a_{22}^{1}=a_{11}^{2}=a_{12}^{2}=0, \quad a_{11}^{1} \neq 0$,

$$
\begin{array}{ll}
g_{11} & =0, \quad g_{12}=a y^{a_{22}^{2}}, \quad g_{22}=0 \quad(a \neq 0), \\
\phi_{1} & =(1 / 2 y) a_{11}^{1}, \quad \phi_{2}=0 .
\end{array}
$$

II(c). $\quad a_{12}^{2}=0, \quad a_{12}^{1}=-\left(a_{22}^{1}\right)^{2} a_{11}^{2} \not \neq 0, \quad a_{11}^{1}=2 a_{22}^{1} a_{11}^{2} \neq 0$,

$$
a_{22}^{2}=1-a_{11}^{2}\left(a_{22}^{1}\right)^{2} \text {, }
$$$$
\phi_{1}=(1 / 2 y) a_{11}^{1}, \quad \phi_{2}=0 \text {. }
$$

$g_{i j}$ as in I(c) with $F(y)=y^{2 a_{12}^{1}}, \quad g(x)=a x^{2}+b x+c$. 
II(d). $\quad a_{12}^{1}=a_{11}^{2}=a_{11}^{1}-a_{12}^{2}=0, \quad a_{11}^{1} \neq 0, \quad a_{22}^{2} \neq 0$,

$$
\begin{aligned}
& g_{11}=\left(2 a_{11}^{1} / a_{22}^{2}\right) g_{12}+c, \quad g_{12}=a a_{11}^{1} \log y+b, \\
& g_{22}=a y^{2 a_{22}^{2}} \\
& (a \neq 0), \\
& \phi_{1}=(1 / y) a_{11}^{1}, \quad \phi_{2}=0 \text {. }
\end{aligned}
$$

$\mathrm{II}\left(\mathrm{d}^{\prime}\right) . \quad a_{12}^{1}=a_{11}^{2}=a_{11}^{1}-a_{12}^{2}=0, \quad a_{11}^{1} \neq 0, \quad \stackrel{2}{a_{22}}=0$,

$$
\begin{aligned}
g_{11} & =a\left(a_{11}^{1}\right)^{2}(\log y)^{2}+2 b a_{11}^{1} \log y+c, \\
g_{12} & =a a_{11}^{1} \log y+b, \quad g_{22}=0, \\
\phi_{1} & =(1 / y) a_{11}^{1}, \quad \phi_{2}=0, \quad a c-b^{2} \neq 0 .
\end{aligned}
$$

$W_{2}$ admitting the three-parameter group

$$
G_{3}=\left[p, 2 x p+y q,\left(x^{2}-4 y^{4} / b^{2}\right) p+\left(x y-2 y^{3} / b\right) q\right]
$$

$$
\begin{aligned}
& \text { III(a). } \quad \Gamma_{11}^{1}=\frac{3 b}{y^{2}}, \quad \Gamma_{12}^{1}=-\frac{9}{y}, \quad \Gamma_{22}^{1}=\frac{12}{b}, \quad \Gamma_{11}^{2}=\frac{b^{2}}{4 y^{3}} \text {, } \\
& \Gamma_{12}^{2}=0, \quad \Gamma_{22}^{2}=-\frac{6}{y} \quad(b \neq 0), \\
& g_{11}=\frac{1}{y^{18}} g(x), \quad g_{12}=\frac{2}{b^{2} y^{15}}\left(g^{\prime}-\frac{3 b}{y^{2}} g\right), \\
& g_{22}=\frac{8}{b^{4} y^{12}}\left(g^{\prime \prime}-\frac{3 b}{y^{2}} g^{\prime}+\frac{9}{2} \frac{b^{2}}{y^{4}} g\right) \text {, } \\
& g(x)=a^{\prime} x^{2}+b^{\prime} x+c, \quad b^{\prime 2}-4 a^{\prime} c \neq 0, \\
& \phi_{1}=\frac{3}{2} \frac{b}{y^{2}}, \quad \phi_{2}=0 .
\end{aligned}
$$

\section{BiBLIOGRAPHY}

1. L. P. Eisenhart, Non-Riemannian geometry, Amer. Math. Soc. Colloquium Publications, vol. 8, 1927.

2. J. Levine, Classification of collineations in projectively and affinely connected spaces of two dimensions, Ann. of Math. vol. 52 (1950) pp. 465-477.

3. T. Y. Thomas, The differential invariants of generalized spaces, Cambridge University Press, 1934, p. 22.

North Carolina State College 